\title{
Group Transfer and Cationic Polymerization of 1-Butadienyloxytrimethylsilane
}

\author{
Tadamichi HiRABAYASHI, Tetsukazu ITOH, and Kenji YoKOTA \\ Department of Materials Science and Engineering, Faculty of Engineering, \\ Nagoya Institute of Technology, \\ Gokiso-cho, Showa-ku, Nagoya 466, Japan
}

(Received March 28, 1988)

\begin{abstract}
As requirement for the synthesis of new C4-type periodic polymers, group transfer polymerization of 1-butadienyloxytrimethylsilane (BdTMS) was examined. Equimolar Benzaldehyde (BAld)- $\mathrm{ZnCl}_{2}$ catalysts in benzene at $20^{\circ} \mathrm{C}$ were effective to give a soluble polymer in organic solvents. The structure of the polymer was revealed by NMR and IR analyses as 1,4-trans addition mode of diene. Dispersities $\left(M_{w} / M_{n}\right)$ of molecular weight distribution evaluated by GPC reduced to about 1.5 , clearly differing from about 3 in the case of $\mathrm{ZnCl}_{2}$ alone. $\mathrm{SnCl}_{4}$ or $\left(\mathrm{C}_{2} \mathrm{H}_{5}\right)_{3} \mathrm{Al}_{2} \mathrm{Cl}_{3}$ without BAld polymerized $\mathrm{BdTMS}$ rapidly, accompanying gelation even at $-78^{\circ} \mathrm{C}$. Living propagation in $\mathrm{BdTMS} / \mathrm{BAld} / \mathrm{ZnCl}_{2}$ system was confirmed by the incremental monomer addition method. In NMR-monitoring the equimolar reaction of BdTMS/BAld catalyzed by $\mathrm{ZnCl}_{2}$ in $\mathrm{C}_{6} \mathrm{D}_{6}$, it was found that an aldol adduct appeared at the first stage and then a formyl group regenerated in the adduct began to react with the monomer.
\end{abstract}

KEY WORDS Periodic Polymer / 1-Butadienyloxytrimethylsilane / Group Transfer Polymerization / Living Polymerization / 1,4-Aldol Adduct / Benzaldehyde / Zinc Chloride /

In order to obtain data on structure-property relationships, we synthesized well-designed copolymers with respect to sequence order, namely periodic copolymers. ${ }^{1-4}$ The alternating copolymerization method catalyzed by Lewis acid was available for this purpose. However, it is almost useless for preparing copolymers of monodisperse distribution of molecular weight, which is required in studying such relationships. Recently new nearly monodisperse polymers could be obtained through 1) the group transfer polymerization method ${ }^{5,6}$ and 2) anionically initiated living polymerization for monomers contain- ing masked functional groups ${ }^{7}$ under rather mild conditions and easier procedures.

A diene derivative with silyl enol ether structure, 1-butadienyloxytrimethylsilane (BdTMS), $\mathrm{CH}_{2}=\mathrm{CH}-\mathrm{CH}=\mathrm{CH}-\mathrm{OSi}\left(\mathrm{CH}_{3}\right)_{3}$, seems to us to be an extremely attractive monomer from the standpoint of preparation of C4-type periodic copolymers, because the monomer must build up a series of polymers as drawn in scheme (1), ( $Z=$ functional group). Furthermore it may be possible to prepare them with a definite molecular size using some suitable catalyst.<smiles>[Z]C(C)CCCCC=CC(C)OC</smiles> 
Though BdTMS and the homolog were synthesized as early as 1974, named "functionalyzed diene" and have been extensively applied $^{8-10}$ in organic syntheses, there is no detailed study in the field of polymerization. This paper describes the polymerization of BdTMS, especially group transfer polymerization in the presence of benzaldehyde-zinc chloride catalysts by which living propagation can occur.

\section{EXPERIMENTAL}

\section{Synthesis of 1-Butadienyloxytrimethylsilane} (BdTMS)

According to Danishevsky procedure, ${ }^{11}$ a benzene solution of trimethylchlorosilane was added to purified crotonaldehyde and triethylamine in benzene with a catalytic amount of zinc chloride $\left(\mathrm{ZnCl}_{2}\right)$, and allowed to react at $60^{\circ} \mathrm{C}$ for $18 \mathrm{~h}$. After removal of the precipitate by filtration in a nitrogen atmosphere, BdTMS was obtainable by distillation as the fraction of $59-60^{\circ} \mathrm{C} / 45 \mathrm{mmHg}$. Yield, $70 \%$. Purity, $99.5 \%$ by gas chromatography. According to ${ }^{1} \mathrm{H},{ }^{13} \mathrm{C}$ NMR and IR data, the most stable geometry of BdTMS is $s$-trans- $Z$-form.

\section{Other Reagents}

Hydrocarbon solvents were purified by the conventional method and dried over sodium, distilled just prior to each polymerization. Benzaldehyde (BAld) was distilled fractionally. $\mathrm{ZnCl}_{2}$ was recrystallized from boiling dioxane solution under nitrogen and dried in vacuo. Commercial stannic chloride $\left(\mathrm{SnCl}_{4}\right)$ and ethylaluminum sesquichloride (EASC) were redistilled under reduced pressure of nitrogen atmosphere.

\section{Polymeriztion}

Typical procedure is as follows. In a dry box under an argon atmosphere, $\mathrm{ZnCl}_{2}$, solvent, BAld and then BdTMS were added in a dry Schlenk tube with a stopcock and a septum cap at $-78^{\circ} \mathrm{C}$, and mixed well at room tem- perature. The tube was sealed by the stopcock and immersed in a thermostated bath. After the monomer was used up, the tube was again brought into the dry box. The solution was carefully separated, using a syringe, from $\mathrm{ZnCl}_{2}$ in the bottom, allowed to evaporate in vacuo, and the polymer was obtained as a highly viscous liquid or soft rubber. Though a thin jellified layer was sometimes formed over $\mathrm{ZnCl}_{2}$, it must not be included in the above solution.

\section{Analyses}

Measurement of ${ }^{1} \mathrm{H}$ and ${ }^{13} \mathrm{C}$ NMR spectra was carried out on a Varian XL-200 or Hitachi $\mathrm{R}-24 \mathrm{~A}$ instrument in benzene- $d_{6}$ or deuterochloroform solution. IR was taken on the JASCO IR-E spectrometer. Gel permeation chromatography (GPC) in tetrahydrofuran (THF) was recorded on a Toyo soda HLG803-D equipped with TSK GMX-, G1000-, G2000-, and G4000-HXL columns in series at $37^{\circ} \mathrm{C}$, by monitroring differential refractometorically. The molecular weights of polymers were calibrated with standard polystyrene samples and then by applying the $Q$-factor method. ${ }^{12} Q$-factor for P(BdTMS), 28.5, was assumed by dividing the molecular weight of monomer by the length of the backbone unit in its streching state.

\section{RESULTS AND DISCUSSION}

\section{Polymerizability of BdTMS}

Polymerization of BdTMS was examined by various catalyst (initiators) and summarized in Table I. Cationic Lewis acid catalysts were effective, while no polymer could be obtained with typical radical and anionic initators $\left(-78^{\circ} \mathrm{C}\right)$. By using $\mathrm{SnCl}_{4}$ and EASC, BdTMS could be polymerized rapidly even at $-78^{\circ} \mathrm{C}$, but was accompanied by serious gelation resulting in almost insoluble polymer in conventional organic solvents. The preferable catalyst was $\mathrm{ZnCl}_{2}$, which gave soluble polymer under mild conditions $\left(20-60^{\circ} \mathrm{C}\right)$. 
Table I. Polymerization of 1-butadienyloxytrimethylsilane (BdTMS) with various catalysts

\begin{tabular}{|c|c|c|c|c|c|c|c|c|c|c|c|}
\hline \multirow{3}{*}{ No. } & \multicolumn{4}{|c|}{ Feed } & \multicolumn{3}{|c|}{ Conditions } & \multicolumn{2}{|c|}{ Polymer yield } & \multirow{2}{*}{\multicolumn{2}{|c|}{$\begin{array}{l}\text { Molecular weight }^{\mathrm{d}} \\
\text { (soluble polymer) }\end{array}$}} \\
\hline & \multirow{2}{*}{$\frac{\text { Monomer }}{\mathrm{mmol}}$} & \multirow{2}{*}{$\frac{\text { Catalyst }}{\mathrm{mmol}}$} & \multicolumn{2}{|c|}{ Initiator $^{\mathbf{a}}$} & \multirow{2}{*}{ Solvent } & \multirow{2}{*}{$\frac{\text { Time }}{\mathrm{h}}$} & \multirow{2}{*}{$\frac{\text { Temp }}{{ }^{\circ} \mathrm{C}}$} & Soluble $^{c}$ & Insoluble & & \\
\hline & & & $\mathrm{mm}$ & & & & & $g(w$ & $t \%)$ & $M_{n} \times 10^{-3}$ & $M_{w} / M_{n}$ \\
\hline 1 & 17.4 & $\mathrm{ZnCl}_{2} \quad 0.59$ & BAld & 0.59 & Benzene & 24 & 20 & $1.94(79)$ & $0.09 \quad(4)$ & 3.10 & 1.57 \\
\hline 2 & 20.3 & $\mathrm{ZnCl}_{2} \quad 0.69$ & BAld & 0.67 & Toluene & 144 & 20 & $2.42(84)$ & $0.16 \quad(5)$ & 2.48 & 1.62 \\
\hline 3 & 17.4 & $\mathrm{ZnCl}_{2} \quad 0.59$ & BAld & 0.59 & Benzene & 24 & 60 & $2.15(88)$ & $0.08 \quad(3)$ & 3.05 & 1.80 \\
\hline 4 & 17.4 & $\mathrm{ZnCl}_{2} \quad 0.59$ & & & Benzene & 24 & 20 & $2.05(84)$ & $0.07 \quad(3)$ & 3.47 & 2.82 \\
\hline 5 & 19.7 & $\mathrm{ZnCl}_{2} \quad 0.65$ & & & Toluene & 144 & 20 & $2.02(72)$ & 0.12 & 4.90 & 3.42 \\
\hline 6 & 20.3 & $\mathrm{ZnCl}_{2} \quad 0.67$ & & & Toluene & 168 & -20 & None & & & \\
\hline 7 & 17.4 & $\mathrm{ZnCl}_{2} \quad 0.59$ & & & $\mathrm{CH}^{\mathrm{b}}$ & 230 & 20 & $1.82(74)$ & $0.20 \quad(8)$ & 3.58 & 3.29 \\
\hline 8 & 8.7 & $\mathrm{SnCl}_{4} \quad 0.29$ & & & Toluene & $\sim 0$ & -78 & - & $1.15(93)$ & & \\
\hline 9 & 12.5 & EASC 0.42 & & & Toluene & 0.5 & -78 & $0.16 \quad(7)$ & $1.24(70)$ & 3.00 & 2.01 \\
\hline 10 & 17.4 & & $\mathrm{BuLi}$ & 0.58 & Toluene & 480 & -78 & None & & & \\
\hline 11 & 20.3 & & AIBN & 0.21 & None & 92 & 120 & None & & & \\
\hline
\end{tabular}

a BAld, benzaldehyde; BuLi, $n$-butyllithium; AIBN, azobisisobutyronitrile.

b $\mathrm{CH}$, cyclohexane.

c See the text.

d Evaluated by GPC method. Detailed description was given in experimental part.

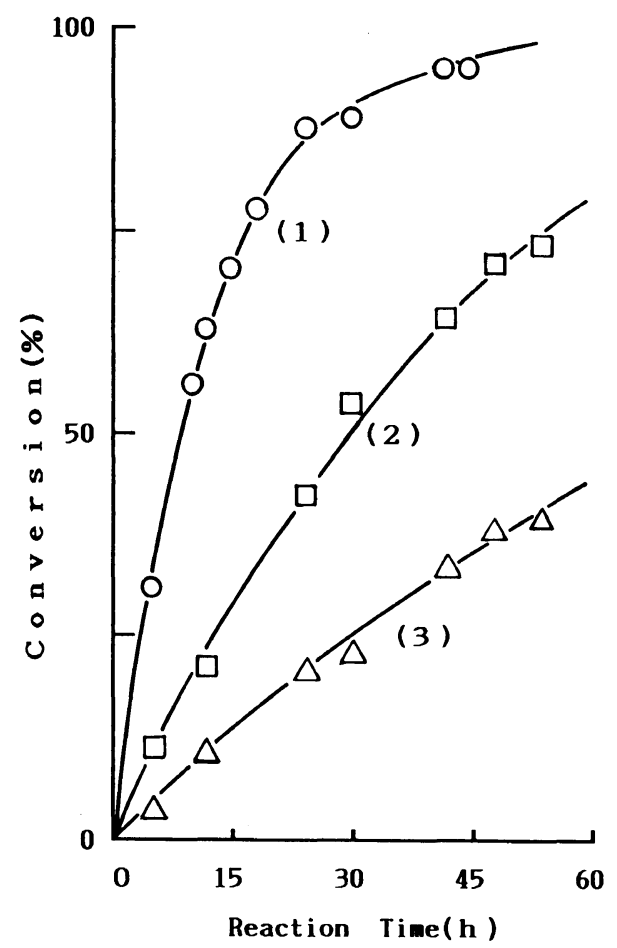

Figure 1. Time-conversion curve in polymerization of BdTMS catalyzed by $\mathrm{ZnCl}_{2}$ alone in (1) benzene, (2) toluene, and (3) cyclohexane at $20^{\circ} \mathrm{C}$. [BdTMS] $=2.05$ $\mathrm{mol} / \mathrm{dm}^{-3} ;[\mathrm{BdTMS}] /\left[\mathrm{ZnCl}_{2}\right]=30$.
The addition of equimolar benzaldehyde (BAld) to $\mathrm{ZnCl}_{2}$ also gave a soluble polymer and make the molecular weight distribution of the polymer narrower than that from $\mathrm{ZnCl}_{2}$ alone. This will be discussed later in detail.

The different polymerization rates were also observed when different hydrocarbon solvents were used. Figure 1 shows time-conversion curves based on ${ }^{1} \mathrm{H}$ NMR-monitoring of the amounts of unreacted monomer.

\section{Structure of Soluble Polymer}

Figures 2, 3, and 4 show ${ }^{1} \mathrm{H},{ }^{13} \mathrm{C}$ NMR and IR spectrum of the soluble polymer, respectively. Assignments of these spectrum demonstrated that the polymer has the structure essentially induced by 1,4-trans addition of diene. No signal due to residual vinyl group caused by 1,2-addition, which often occurred in diene polymerization, was observed. A few small signals nearby $125-130 \mathrm{ppm}$ in ${ }^{13} \mathrm{C}$ NMR spectrum (Figure 3 ) should originate in BAld, which must be incorporated into the polymer. Trimethylsilyloxy group remained without disproportionation, which was also 


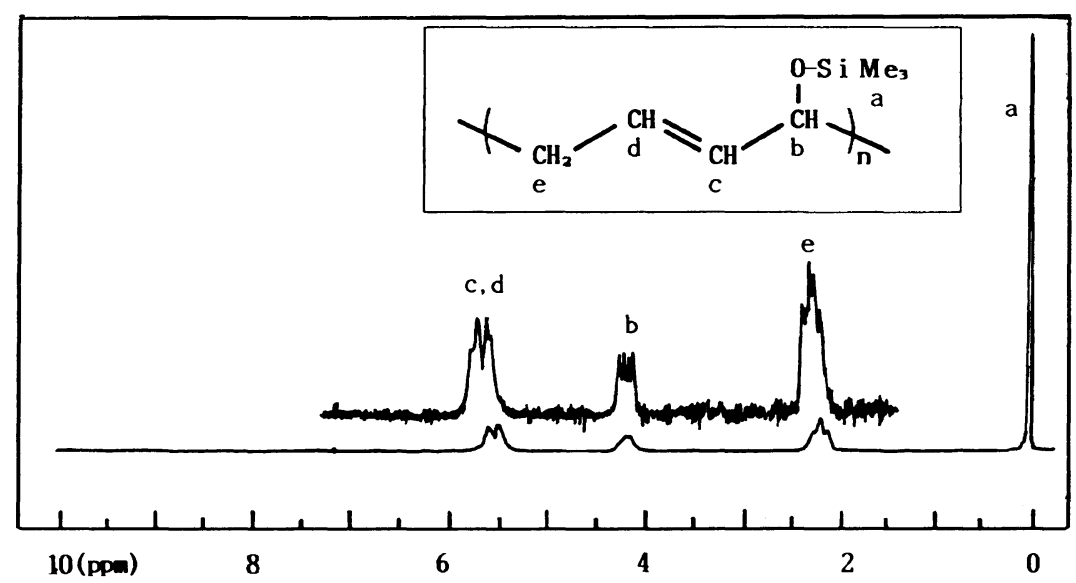

Figure 2. ${ }^{1} \mathrm{H}$ NMR $(200 \mathrm{MHz})$ spectrum of BdTMS polymer (sample, No. 1 in Table I) in $\mathrm{CDCl}_{3}$, and assignments.

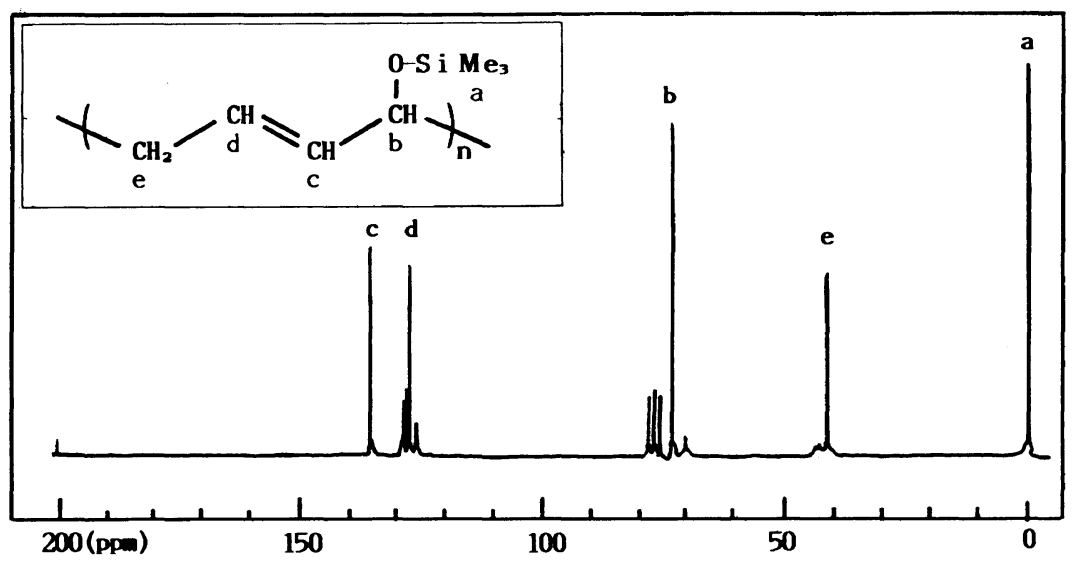

Figure 3. ${ }^{13} \mathrm{C}$ NMR spectrum of BdTMS polymer (sample, No. 1 in Table $\mathrm{I}$ ) in $\mathrm{CDCl}_{3}$, and assignments.

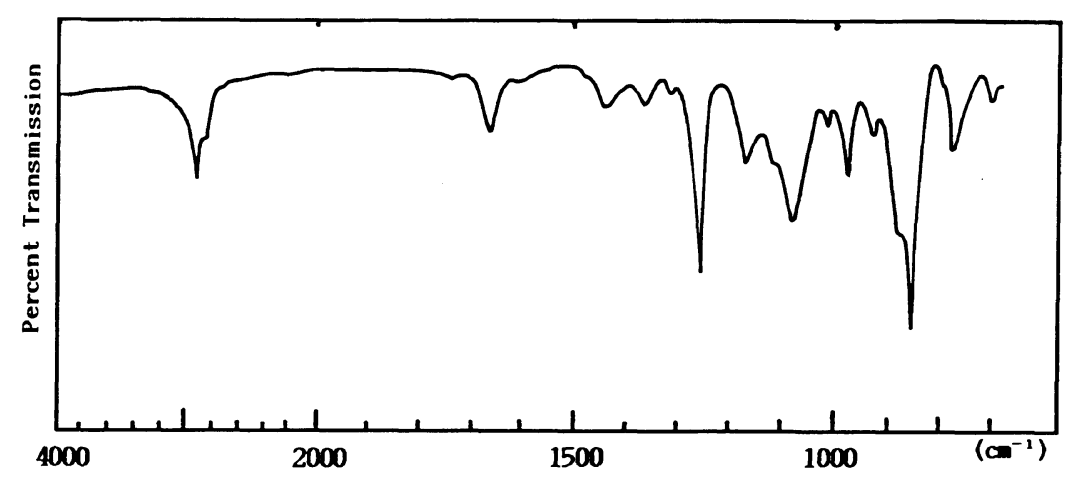

Figure 4. IR spectrum of BdTMS polymer (sample, No. 1 in Table I) on KBr. 
comfirmed by elemental analysis in accordance with $\mathrm{C} / \mathrm{H}=1 / 2$. However, for the gelated polymers, elemental analysis data $(\mathrm{C} / \mathrm{H}=1 / 1.6-$ 1.8) considerably deviated from the theoretical value $(1 / 2)$ and suggested that trimethylsilyl group must be eliminated from those polymers and cross-linking must be caused by such elimination reaction. The structure of polymer end will be discussed in a latter section.

The difference in the chemical structure between the soluble polymers produced by BAld$\mathrm{ZnCl}_{2}$ system and by $\mathrm{ZnCl}_{2}$ alone was hardly discernible. However, the former had a narrower molecular weight distribution than the latter. This suggests that the former has living character in propagation, though the conditions required for the strictly defined "living polymerization" have been unsatisfied as yet.

\section{Confirmation of Living Propagation in Benzal- dehyde-Zinc Chloride System}

An incremental monomer addition method was carried out to confirm that the polymer end was active (living) to an additional monomer, as illustrated by GPC charts in Figure 5. A solution of $\mathrm{BdTMS} / \mathrm{BAld} / \mathrm{ZnCl}_{2}$ $(=26.9 / 1.0 / 1.0)$ in benzene- $d_{6}$ allowed polymerization at $20^{\circ} \mathrm{C}$. In a solution picked up through the septum after $23 \mathrm{~h}$ reaction, no monomer was detected by ${ }^{1} \mathrm{H}$ NMR. The sample solution was concentrated and GPC of the resulting polymer was performed, estimating $\bar{M}_{n}=2470, \bar{M}_{w} / \bar{M}_{n}=1.56$ [Figure 5/(1)]. When the additional monomer was poured into the original mixture through the septum, polymerization occurred again and $M_{n}$ of the polymer increased till the monomer was used up again. GPC charts of the samples prepared in the same manner in the course of the second step are presented in Figure 5/(2-1)(2-3). It could be read that a) $\bar{M}_{w} / \bar{M}_{n}$ was kept in a range of $1.5-1.6$ throughout the first and second steps, b) no new GPC-peaks appeared in lower molecular weight region than the first state, and c) the polymer attained finally $M_{n}=4660$, meaning that each polymer

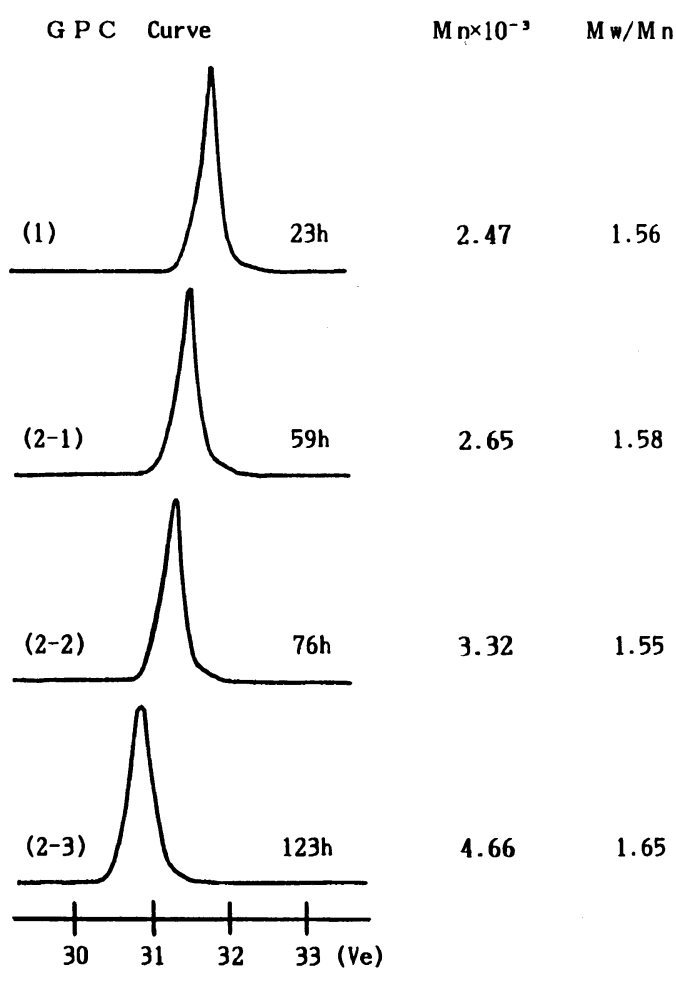

Figure 5. Change of molecular weight of the BdTMS polymers through the incremental monomer addition procedure. (1) After the first polymerization: BdTMS, 29.0; BAld, 1.08; and $\mathrm{ZnCl}_{2}, 1.07 \mathrm{mmol}$ in $\mathrm{C}_{6} \mathrm{D}_{6}$ at $20^{\circ} \mathrm{C}$. (2-1)-(2-3) In the course of the second polymerization in which $17.4 \mathrm{mmol}$ of BdTMS were added to the first. As for GPC conditions, see the text.

increased by about 2190 with respect to $M_{n}$ during the second step, (i.e., 15.4 as the degree of polymerization). It may be worthwhile to note [added BdTMS]/[BAld] =17.4/1.08. These results would be explained satisfactorily only when the second polymerization could initiate at the active end of the first polymer and propagation could proceed in the living manner. Consequently, the next question is why this system shows such living behaviour.

Initiation of Polymerization: Transfer of Trimethylsilyl Group

In order to clarify the initiation reaction, the equimolar raction of BdTMS and BAld in the presence of $\mathrm{ZnCl}_{2}$ was investigated by means 


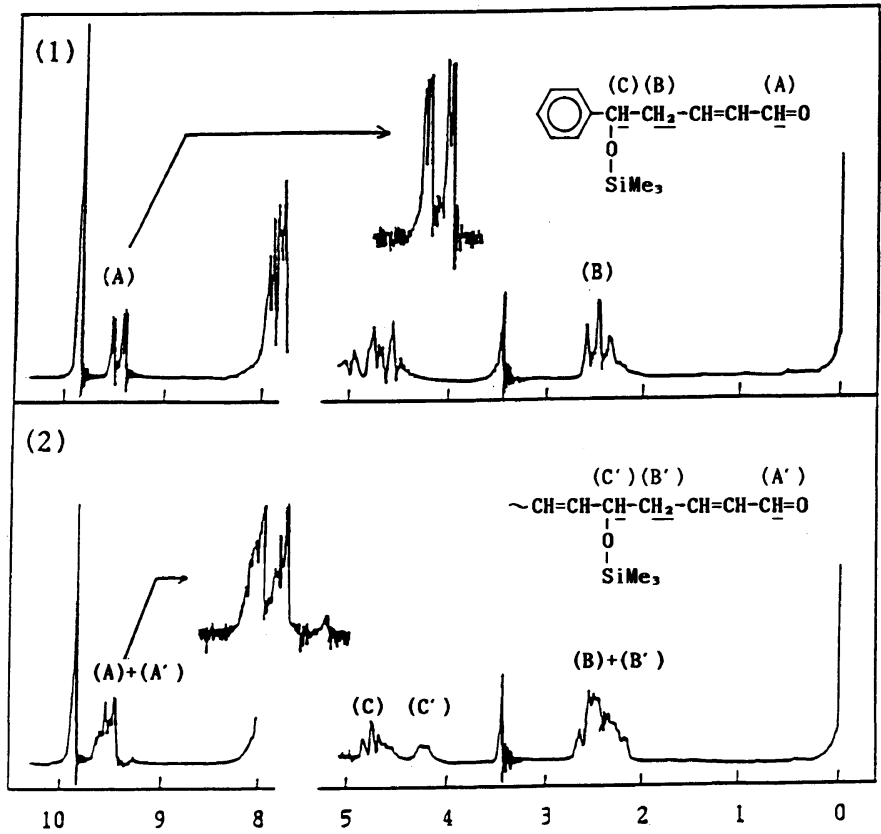

Figure 6. ${ }^{1} \mathrm{H}$ NMR $(60 \mathrm{MHz})$ spectra of the equimolar mixture of BdTMS and BAld in the presence of $\mathrm{ZnCl}_{2}$ in benzene- $d_{6}$ after (1) $15 \mathrm{~min}$ and (2) $90 \mathrm{~min}$. Initial concentration ratio, [BdTMS]/[BAld]/[ $\left.\mathrm{ZnCl}_{2}\right]=$ $5 / 5 / 1$. As an internal standard, dioxane $(\delta 3.5)$ was used.

of ${ }^{1} \mathrm{H}$ NMR. BdTMS, BA1d, and $\mathrm{ZnCl}_{2}$ $(=5 / 5 / 1)$ in benzene- $d_{6}$ were diectly mixed in a NMR tube and the sample was immediately observed in the probe $\left(35^{\circ} \mathrm{C}\right)$. The change of ${ }^{1} \mathrm{H}$ NMR spectra is shown in Figure 6. In the absence of $\mathrm{ZnCl}_{2}$, however, no change could be found even after one day.

At the initial stage, three new absorptions at $\delta=9.50(\mathrm{~d}-\mathrm{d}),[(\mathrm{A})$ in Figure 6(1)], 2.50 (t), [(B)] and near $4.8 \mathrm{ppm}$ appeared in the ratio of $1: 2:(1)$, respectively. The first one was distinct from the formyl proton $(9.92, \mathrm{~s})$ in BAld and should be assigned to the formyl proton on $-\mathrm{CH}=\mathrm{CH}-\mathrm{C} \underline{\mathrm{H}}=\mathrm{O}$ moiety although corresponding olefinic protons were obscure because of overlapping with protons due to the unreacted monomer. The last two were assignable to methylene and methine protons on $=\mathrm{CH}-\mathrm{CH}_{2}-\mathrm{CH}\left(\mathrm{OSiMe}_{3}\right)-\mathrm{C}_{6} \mathrm{H}_{5}$ moiety, respectively. Two kinds of methyl protons on silicon were also observed with so close a chemical shift. The intensity of signals due to monomer decreased with time. All these indicated that the trimethylsilyl group transferred from monomer onto aldehyde oxygen with the assistance of $\mathrm{ZnCl}_{2}$, and 1,4-aldol adduct was formed, as shown in eq 2 .<smiles>C=CC=COCC</smiles>

However, the aldol adduct was not entirely formed in this stage. The intensity ratio of $I_{9.92} / I_{9.50}$ varied from $73.0 / 27.0$ (15 min after mixing), $65.5 / 34.5$ (45 $\mathrm{min})$, to $62.0 / 38.0$ (90 min) and hereinafter was almost constant. To complicate matters, the adduct itself had a formyl group which must be sensitive to the above-mentioned reaction. In fact, the secondary changes in NMR spectrum began to be 
observed after about $30 \mathrm{~min}$. The sharp doublet signal at 9.50 changed to squat halves. The third peak due to trimethylsilyl protons was closely but clearly found between the first two. The typical triplet signal at 2.50 was also deformed with signals from the higher magnetic field side. A new signal $\left(\mathrm{C}^{\prime}\right)$ appeared at $\delta 4.22$ and must be assignable to methine proton in $2 \mathrm{CHOSi}\left(\mathrm{CH}_{3}\right)_{3}$ moiety which is remote from the phenyl group. Therefore, this chemical shift is substantially the same with that of polymer.

However, the total amounts of formyl group never varied during measurements based on the relative intensity for the internal standard (dioxane, 83.50$)$. When the monomer in NMR sample was used up ( $\sim 120 \mathrm{~min})$, this spectrum could be assigned as a mixture of the first adduct, oligomers (polymers) of BdTMS, and unreacted BAld.

Thus we ascertained that 1) the 1,4-dldol addition caused by the transfer of trimethylsilyl group to formyl oxygen in BdTMS was the real initiation reaction, 2) crotonaldehyde- type formyl group reproduced in the adduct could rather readily undergo the similar addition reaction with BdTMS, and 3) the ends of polymer obtained from $\mathrm{BAld} / \mathrm{ZnCl}_{2}$ system must consist of a phenyl group and formyl group which was always reactive to the monomer.

The addition of equimolar BdTMS to the above NMR sample after $2 \mathrm{~h}$, (i.e., BdTMS/ $\mathrm{BAld} / \mathrm{ZnCl}_{2}$ was reset as $\left.10 / 5 / 1\right)$, only changed the intensity ratio of $I_{9.92} / I_{9.50}$ from $61.1 / 38.9$ to $59.8 / 40.2$. The signals due to oligomer (polymer) gradually increased. It was again demonstrated that the added monomer predominantly reacted at the formyl group in polymer in spite of the presence of unreacted BAld. Therefore, active $\mathrm{ZnCl}_{2}$ species might be restricted near the polymer end.

\section{Polymerization Mechanism of BdTMS Cata- lyzed by Benzaldehyde- $\mathrm{ZnCl}_{2}$ \\ On the basis of results described above, we propose the polymerization mechanism catalyzed by BAld- $\mathrm{ZnCl}_{2}$ as eq 3 .}

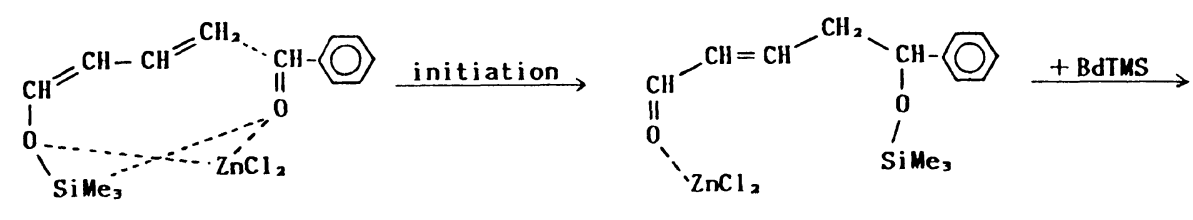

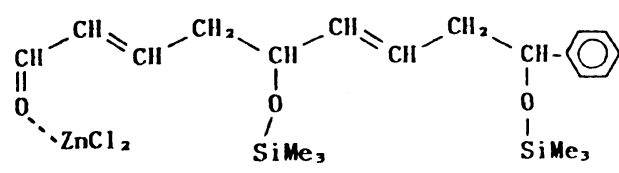

It may be possible to speculate alternative interaction modes of $\mathrm{ZnCl}_{2}$. However, it is most reasonable to imagine an 8-membered transition state in order that the TMS-group transfer from a monomer onto formyl oxygen atom can give 1,4-aldol adduct. Such a situation can be permitted by the considerably large bond radius of $\mathrm{Si}$ and $\mathrm{Zn}$. In contrast with vinyloxytrimethylsilane, ${ }^{6}$ the 6 -membered transition stage cannot be preferred because of the $s$-trans- $Z$ structure of the monomer. In

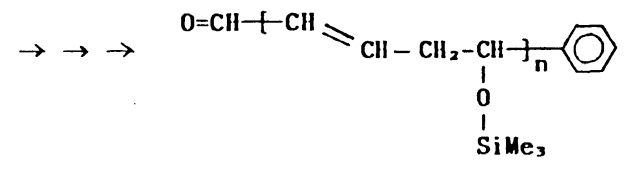

fact, there was little evidence for 1,2-addition.

It is proper that this group transfer polymerization requires room temperature or thereabout, but not higher, since both the initiation and propagation were the so-called smart organic reactions. In spite of the living propagation, the molecular weight distribution was not so remarkably narrow. To explain this disadventage, it should be remembered that the intiation reaction could not occur rapidly and, moreover, was forced to compete with 
propagation under the given conditions. There may be place for further improvement in this system.

Hydrolyses without gelation and other desilylation procedures followed by introducing some suitable functional group in this polymer are in progress and will be reported in following paper.

\section{REFERENCES}

1. K. Yokota and T. Hirabayashi, Macromolecules, 14, 1613 (1981).

2. K. Yokota, T. Kougo, and T. Hirabayashi, Polym. J., 15, 349 (1983).

3. K. Yokota, T. Kougo, and T. Hirabayashi, Polym. J., 15, 891 (1983).
4. K. Yokota and T. Hirabayashi, Polym. J., 17, 991 (1985).

5. O. W. Webster, W. R. Hertler, D. Y. Sogah, W. B. Franham, and Rajanbabu, J. Am. Chem. Soc., 105, 5701 (1983); see also a summarized article, for example, Macromolecules, 20, 1473 (1987).

6. a) R. Pariser, Japan-US Polymer Symposium, 1985, p 20.

b) D. Y. Soga and O. W. Webster, Macromolecules, 19, 1775 (1986).

7. A. Hirao, K. Takenaka, K. Yamaguchi, S. Nakahama, and N. Yamazaki, Polymer, 314 (1983); see also subsequent papers.

8. K. Yamamoto, S. Suzuki, and J. Tsuji, Chem. Lett., 649 (1978).

9. S. Danishevsky, Acc. Chem. Res., 14, 400 (1981).

10. R. R. Schmidt, Acc. Chem. Res., 19, 250 (1986).

11. S. Danishevsky and T. Kitahara, J. Org. Chem., 40, 538 (1975).

12. D. J. Harmon, J. Polym. Sci. C, 8, 243 (1965). 